\title{
Bruxismo y parasitosis intestinal en niños de 4 a 6 años de edad en La Brea (Talara, Piura) Perú
}

\author{
Bruxism and intestinal parasites in 4-6 years old children in La Brea (Talara, Piura) Peru
}

Cindy Baldeón Sifuentes ${ }^{1, a}$, César del Castillo López ${ }^{1, b, c,}$ José Antonio Balarezo Razzeto $^{2, b, d}$, Alexis Evangelista Alva ${ }^{1, b, e}$, Pablo Sánchez Borjas ${ }^{3, b}$, José Luis Velásquez Sánchez ${ }^{4, f}$

\section{RESUMEN}

La prevalencia del bruxismo es variada, sin diferencias por sexos, pero si por edades; más frecuente en jóvenes, disminuyendo en niños con mayor edad. La parasitosis intestinal tiene distribución amplia en la población mundial, constituye un problema de salud pública, que afecta a la quinta parte la población mundial, y en Sudamérica la proporción es aún mayor. Algunos autores mencionan al bruxismo como sintomatología asociada con parasitosis intestinal. Sin embargo, la relación bruxismo-parasitosis aún no ha sido establecida. Objetivos: Evaluar la asociación entre bruxismo y parasitosis intestinal en niños de 4 a 6 años de edad, en el distrito de La Brea (Talara, Piura) Perú. Material y métodos: Estudio transversal, analítico, la muestra estuvo formada por 92 niños de 4 a 6 años de edad, de 10 Centros Educativos. Se obtuvo el Consentimiento Informado de los padres y el registro si el niño rechinaba los dientes. Se analizaron muestras seriadas de heces en el Puesto de Salud local por examen directo copro-parasitológico. Resultados: El 65,22\% de los niños tuvo bruxismo, este fue más frecuente en niños de 5 años de edad. El 30.4\% de los niños tuvieron resultados positivos de parasitosis intestinal, siendo mayor el porcentaje de niñas afectadas. Los parásitos más frecuentes fueron: Giardia lamblia y Áscaris lumbricoides. No se encontró Enterobius vermicularis. No se observó diferencias significativas entre parasitosis y tipo de parásito encontrado, según sexo. Conclusiones: No se halló asociación entre parasitosis y bruxismo.

\section{PALABRAS CLAVE: Bruxismo, parasitosis intestinales, niños. (DeCS, BIREME)}

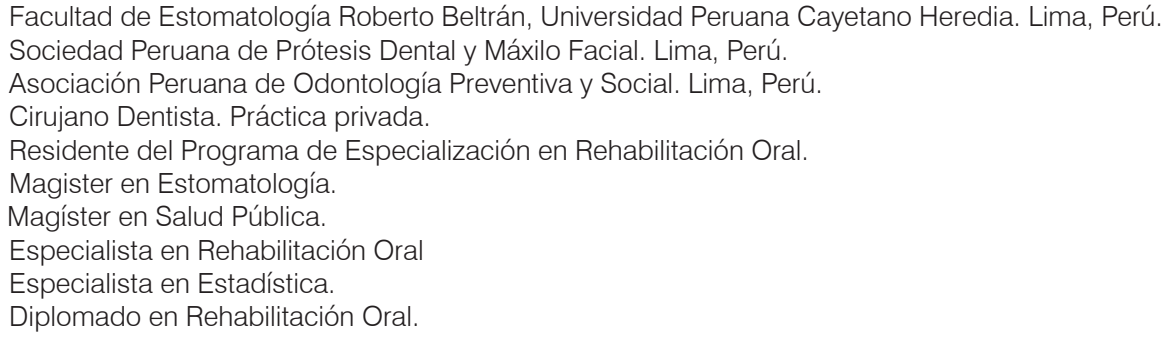




\section{SUMMARY}

Population frequency of bruxism is varied, without differences by gender, but different by age. It is more frecuent in young people and it decreases in older children. Intestinal parasitosis is widely distributed in world population, is a public health problem affecting a fifth of the world population, although this proportion increase in South America. Some studies have mentioned the bruxism as a symptom associated with intestinal parasitosis. However, the association bruxism-parasitosis has not yet been established. Objectives: The aim of the study was to evaluate the association between bruxism and intestinal parasitosis in children aged 4-6 years in the district La Brea (Talara, Piura) Perú. Methods: A cross-sectional study in 92 children, both sexes, 4-6 years old, in 10 schools. Informed parental consent was obtained. Parents were asked to check on whether their child grinded his teeth. Serial stool samples were analyzed at the local health Center with copro-parasitological direct examination. Results: $65.22 \%$ of the children had bruxism, most frequent at 5 years of age and more common in girls. The 30.4\% of children tested positive for intestinal parasites, most frequently in girls than in boys. The most frequently parasites were Giardia lamblia and Ascaris lumbricoides. No Enterobius vermicularis was found. Differences between the presence of parasites by sex was not found. Conclusions: No association between parasitism and bruxism was found.

\section{KEYWORDS: Bruxism, parasitosis diseases, children. (MeSH, NLM)}

\section{INTRODUCCIÓN}

El bruxismo es definido en el Glosario de Términos Prostodónticos (2005), como el desgaste parafuncional de los dientes y como un hábito involuntario rítmico el cual puede llevar a un trauma oclusal. Afecta tanto a niños como a adultos y necesita tratamiento $(1,2)$. Para la Academia Americana de Dolor Orofacial (2008), es una actividad parafuncional, diurna o nocturna, que incluye el apretamiento, rechinamiento y desgaste de los dientes, considerada como la actividad parafuncional más perjudicial del sistema estomatognático, que ocasiona desgaste dentario, lesiones del tejido periodontal y daño articular y/o muscular (3). La Academia Americana de Medicina del Sueño (2005) define el bruxismo nocturno como un desorden relacionado al sueño, caracterizado por el rechinamiento o el apretamiento de los dientes durante el sueño, usualmente asociado con la fase de despertar (4).

La prevalencia del bruxismo en niños es muy variada, reportándose en una población pediátrica de Hong Kong (5) 8,5\% y en niños americanos 38\% (6); en Sudamérica se han encontrado porcentajes aún mayores, así en Brasil 43\% (7) y en Perú (Buenos Aires, Morropón, Piura) 69,8\% (8). No encontrándose diferencias por sexo; pero si, según la edad; siendo más frecuente en jóvenes y disminuyendo en edades mayores (9).
Los signos y síntomas del bruxismo incluyen desgaste dental, abfracciones, movilidad, fracturas dentales, trauma oclusal, fatiga y/o dolor muscular, ruidos y/o dolor articular y cefalea (10). Los síntomas en niños pueden persistir hasta la adultez y llevar a problemas de la articulación temporomandibular, músculos faciales, pérdida de estructura dentaria, problemas periodontales e incluso pérdida de piezas dentarias $(11,12)$.

La etiología del bruxismo ha sido definida como multifactorial, siendo regulada por el sistema nervioso central e influenciada periféricamente. Es decir, que los hábitos bucales, desórdenes temporomandibulares, maloclusiones, ansiedad, personalidad y stress -entre otros- podrían influenciar la presencia del bruxismo periféricamente, ya que actúan como estímulo del sistema nervioso central, el cual reacciona alterando la neurotransmisión de dopamina, siendo la respuesta el apretamiento y rechinamiento de los dientes $(13,14)$. En la etiología del bruxismo nocturno se consideran factores neurológicos, estímulos periféricos y elementos psicogenéticos. Se ha sugerido también, que algunos medicamentos podrían estar relacionados al bruxismo (13).

Algunas condiciones sistémicas han sido reportadas como factores de riesgo del bruxismo, tales como: alergias, problemas endocrinos, desnutrición y desórdenes gastrointestinales, dentro de éstos últimos, la parasitosis intestinal (15). 
La parasitosis intestinal es reconocida como causa de enfermedades en el hombre, considerándose su presencia un indicador de pobre desarrollo social en una región o país (1). Su distribución es amplia en la población mundial y constituye un problema de salud pública, pues afecta a la quinta parte la población mundial; siendo Sudamérica la región con una gran proporción de población afectada (16).

Las infecciones parasitarias intestinales son producidas por protozoarios y helmintos, cuya vía de infección es la digestiva y en algunos casos la cutánea (6). Causan numerosos síntomas entre los cuales se encuentra el bruxismo. Los protozoos más comunes son: Giardia lamblia y Entamoeba coli. Los helmintos más frecuentes son: nematodos (Trichuris trichiura, Enterobius vermicularis, Áscaris lumbricoides), trematodos (Onchocerca volvulus, Schistosoma haematobium) y cestodos (Hymenolepis nana, Taenia saginata, Taenia solium) $(17,18)$.

El Enterobius vermicularis, conocido también como oxiuro, se encuentra en poblaciones de todo el mundo. Es más común en las regiones templadas, en condiciones de hacinamiento; por ejemplo, en centros de cuidado diurnos y colegios (19). Clínicamente pueden ser asintomáticos o sintomáticos. Cuando su número es alto puede existir prurito anal, perianal, nasal y dolores abdominales. Puede asociarse también a nerviosismo diurno, con modificaciones en la conducta, agitación nocturna, insomnio y bruxismo $(17-21)$.

Ledesma y Fernández (22) encontraron al Enterobius vermicularis como el segundo parásito de mayor prevalencia, observando un $44 \%$ de preescolares y escolares infectados de la zona periurbana de Resistencia (Chaco, Argentina).

Otros parásitos intestinales (protozoarios y helmintos) tienen prevalencia variada en el mundo y se trasmiten principalmente por vía fecal-bucal, por manejo inadecuado de alimentos o bebidas, o a través de insectos como moscas. Pueden ser asintomáticos o estar asociados a diversos síntomas y enfermedades presentando el paciente dolores y cólicos abdominales, cefaleas, sintomatología neurológica, anorexia, diarreas, anemia y otras molestias mal definidas (21).

En el Perú, la parasitosis intestinal tiene alta prevalencia, determinándose que uno de cada tres peruanos es portador de uno o más parásitos en el intestino. $\mathrm{Su}$ distribución varía según regiones geográficas, con predominio de helmintos en la selva y de protozoos en la costa, con diferencias entre la población urbana y rural (23). Delgado (8) el año 2002 en Buenos Aires (Morropón, Piura), encontró una prevalencia de parasitosis de 52,39\% en preescolares de un Centro Educativo Inicial; correspondiendo el $56,67 \%$ al sexo masculino y el $48,48 \%$ al sexo femenino.

Creencias populares y algunos estudios $(15,21,24,25)$ sugieren la existencia de una posible asociación entre bruxismo y la presencia de parásitos intestinales; pero otros estudios $(8,26,27)$ no han encontrado tal asociación.

Debido a su importancia en salud pública, tanto de parasitosis intestinal como de bruxismo, el objetivo del presente estudio fue, evaluar la posible asociación entre bruxismo e infección por parásitos intestinales en niños de 4 a 6 años de edad, del distrito de La Brea (Talara, Piura, Perú).

\section{MATERIAL Y MÉTODOS}

Estudio transversal, analítico, en una población de 396 niños de 4 a 6 años de edad, de ambos sexos, de 10 Centros Educativos estatales y particulares en La Brea, Provincia de Talara, Departamento de Piura (Perú). La muestra estuvo constituida por 92 niños quienes cumplieron los siguientes criterios de selección: a) niños de 4 a 6 años de edad, b) consentimiento de los padres o tutores, c) dentición decidua completa. Se excluyeron a aquellos que presentaron: a) problemas de comportamiento, b) alguna enfermedad sistémica, c) diarreas, d) tratamiento antiparasitario reciente, e) Caries de Infancia Temprana, y f) aquellos en los que no se completó la serie de exámenes de tres muestras de heces.

Se realizaron las coordinaciones necesarias y se obtuvieron los permisos correspondientes de las autoridades de los Centros Educativos y de la Posta de Salud JOSPICE-Casa San José, para llegar a los padres de familia para la selección de los niños y para el examen copro-parasitológico de muestras de heces.

En cada Centro Educativo se realizó un taller informativo a los padres o tutores de los niños, acerca del estudio para obtener el Consentimiento para que 
sus hijos participaran en el estudio. Se solicitó a los padres de familia que observaran durante siete días si sus hijos rechinaban los dientes durante la noche y se les entregó envases estériles para la recolección de muestras de heces en tres días consecutivos, debiendo entregarlas en la posta de salud para los exámenes parasitológicos. El personal del laboratorio realizó el examen directo coproparasitológico seriado de las muestras de heces, en láminas porta objetos utilizando gotas de solución de suero fisiológico y lugol, mediante la observación directa al microscopio (10x y 40x) para detectar la presencia y tipo de parásitos. Los resultados fueron entregados a los investigadores del presente estudio y al Centro Educativo, para derivar a los niños con diagnóstico de parasitosis a la posta para su tratamiento.

La evaluación dental de cada niño, se realizó, en un ambiente de su Centro Educativo, con el niño sentado, luz natural, espejo bucal y explorador.

Se procesó la frecuencia absoluta y relativa de niños según edad, sexo, presencia de bruxismo, y presencia de parasitosis. Se realizaron pruebas de asociación entre bruxismo y parasitosis mediante la Prueba Chi ${ }^{2}$.

Tabla 1. Características de la muestra (n: 92).

\begin{tabular}{lcc}
\hline & $\mathbf{N}^{\mathbf{0}}$ & $\mathbf{\%}$ \\
\hline Centro Educativo & & \\
Cristo Rey & 8 & 8,70 \\
J.A. Encinas & 19 & 20,65 \\
San Antonio & 5 & 5,43 \\
Juan Pablo II & 14 & 15,22 \\
Santa María Reyna & 6 & 6,52 \\
La Capilla & 3 & 3,26 \\
La Casa del Saber & 12 & 13,04 \\
Primeros Frutos & 8 & 8,70 \\
Trilce Fe y Esperanza & 3 & 3,26 \\
Francisco Bolognesi & 14 & 15,22 \\
Edad & & \\
4 años & 42 & 45,65 \\
5 años & 39 & 42,39 \\
6 años & 11 & 11,96 \\
Sexo & & \\
Masculino & 45 & 48,91 \\
Femenino & 47 & 51,09 \\
\hline
\end{tabular}

Para el estudio se contó con la aprobación del Comité Institucional de Ética de la Universidad Peruana Cayetano.

\section{RESULTADOS}

De los 92 niños seleccionados, 42 (45,65\%) fueron de 4 años de edad, 39 (42,39\%) de 5 años y 11 $(11,96 \%)$ de 6 años; 45 fueron niños $(48,91 \%)$ y 47 niñas $(51,09 \%)$ (Tabla 1$)$.

El $65,22 \%$ de la muestra tenía el hábito de bruxismo, siendo más frecuente en niños de 5 años de edad (71,8 \% de 39), seguido de 4 años (61,9\% de 42$)$ y de los de 6 años $(54,5 \%$ de 11$)$. Respecto al sexo, en las niñas la frecuencia fue mayor $(74,5 \%$ de 47$)$, que en los niños $(55,6 \%$ de 45$)$. No existió diferencia estadísticamente significativa entre bruxismo según edad y sexo (Tabla 2).

Se observó que 28 niños de la muestra total $(30,4 \%)$ sufrían de parasitosis intestinal, de los cuales el 60,7\% tenía 4 años de edad, el 32,1\% 5 años y el 7,1\% 6. El parásito con mayor presencia fue: Giardia Lamblia en 14 niños (50\%), seguido de Áscaris lumbricoides en 9 niños $(32,1 \%)$. No se encontró el tipo Enterobius vermicularis. Tampoco se encontraron diferencias significativas entre presencia de parasitosis o tipo de parásito, según edad de los niños (Tabla 3).

Tabla 2. Frecuencia de bruxismo según edad y sexo.

\begin{tabular}{ccccccc}
\hline & \multicolumn{3}{c}{ BRUXISMO } & & \\
& \multicolumn{2}{c}{ No } & \multicolumn{2}{c}{ Si } & \multicolumn{2}{c}{ Total } \\
& n & \% & n & \% & n & \% \\
\hline Edad & & & & & & \\
4 años & 16 & 38,10 & 26 & 61,90 & 42 & 45,65 \\
5 años & 11 & 28,20 & 28 & 71,80 & 39 & 42,39 \\
6 años & 5 & 45,50 & 6 & 54,50 & 11 & 11,96 \\
Total & 32 & 34,78 & 60 & 65,22 & 92 & 100,00 \\
p $\geq 0,05$ & & & & & & \\
& & & & & & \\
Sexo & & & & & & \\
Masculino & 20 & 44,4 & 25 & 55,6 & 45 & 48,9 \\
Femenino & 12 & 25,5 & 35 & 74,5 & 47 & 51,1 \\
Total & 32 & 34,8 & 60 & 65,2 & 92 & 100,0 \\
p $\geq 0,05$ & & & & & & \\
$0,88<$ OR $<6,26$ & & & & & & \\
\hline
\end{tabular}


Tabla 3. Frecuencia de parasitosis y tipo de parásito intestinal según edad.

\begin{tabular}{|c|c|c|c|c|c|c|c|c|}
\hline \multirow[t]{3}{*}{ PARASITOSIS } & \multicolumn{6}{|c|}{ EDAD } & \multicolumn{2}{|c|}{ Total } \\
\hline & \multicolumn{2}{|c|}{4 años } & \multicolumn{2}{|c|}{5 años } & \multicolumn{2}{|c|}{6 años } & \multirow[b]{2}{*}{$\mathbf{n}$} & \multirow[b]{2}{*}{$\%$} \\
\hline & $\mathbf{n}$ & $\%$ & $\mathbf{n}$ & $\%$ & $\mathbf{n}$ & $\%$ & & \\
\hline \multicolumn{9}{|l|}{ Parasitosis } \\
\hline Sin parasitosis & 25 & 39,1 & 30 & 46.9 & 9 & 14.1 & 64 & 69.6 \\
\hline Con parasitosis & 17 & 60,7 & 9 & 32.1 & 2 & 7.1 & 28 & 30.4 \\
\hline Total & 42 & 45,7 & 39 & 42.4 & 11 & 12.0 & 92 & 100.0 \\
\hline \multicolumn{9}{|l|}{$\mathrm{p} \geq 0,05$} \\
\hline \multicolumn{9}{|l|}{ Tipo de Parásito } \\
\hline Áscaris lumbricoides & 6 & 21,4 & 3 & 10,7 & 0 & 0,0 & 9 & 32,1 \\
\hline Hymenolepsis nana & 1 & 3,6 & 0 & 0,0 & 0 & 0,0 & 1 & 3,6 \\
\hline Quiste Giardia lambilia & 8 & 28,6 & 5 & 17,9 & 1 & 3,6 & 14 & 50,0 \\
\hline Quiste Entamoeba coli & 0 & 0,0 & 1 & 3,6 & 1 & 3,6 & 2 & 7,1 \\
\hline Tenia $S$. & 1 & 3,6 & 0 & 0,0 & 0 & 0,0 & 1 & 3,6 \\
\hline Trichuris trichura & 1 & 3,6 & 0 & 0,0 & 0 & 0,0 & 1 & 3,6 \\
\hline Larva Enterobius verniculares & 0 & & 0 & & 0 & & 0 & 0,0 \\
\hline Total & 17 & 60,7 & 9 & 32,1 & 2 & 7,1 & 28 & 100,0 \\
\hline $\mathrm{p} \geq 0,05$ & & & & & & & & \\
\hline
\end{tabular}

Tabla 4. Frecuencia de parasitosis y tipo de parásito intestinal según sexo.

\begin{tabular}{|c|c|c|c|c|c|c|}
\hline & \multicolumn{4}{|c|}{ SEXO } & \multicolumn{2}{|c|}{ Total } \\
\hline & \multicolumn{2}{|c|}{ Masculino } & \multicolumn{2}{|c|}{ Femenino } & \multirow[b]{2}{*}{$\mathbf{n}$} & \multirow[b]{2}{*}{$\%$} \\
\hline & $\mathbf{n}$ & $\%$ & n & $\%$ & & \\
\hline \multicolumn{7}{|l|}{ Parasitosis } \\
\hline Sano & 35 & 54,7 & 29 & 45,3 & 64 & 69,6 \\
\hline Con parasitosis & 10 & 35,7 & 18 & 64,3 & 28 & 30,4 \\
\hline Total & 45 & 48,9 & 47 & 51,1 & 92 & 100,0 \\
\hline \multicolumn{7}{|l|}{$\mathrm{p} \geq 0,05$} \\
\hline \multicolumn{7}{|l|}{ Tipo de Parásito } \\
\hline Áscaris lumbricoides & 4 & 14,3 & 5 & 17,9 & 9 & 32,1 \\
\hline Hymenolepsis nana & 0 & 0,0 & 1 & 3,6 & 1 & 3,6 \\
\hline Quiste Giardia lamblia & 4 & 14,3 & 10 & 35,7 & 14 & 50,0 \\
\hline Quiste Entamoeba coli & 2 & 7,1 & 0 & 0,0 & 2 & 7,1 \\
\hline Tenia $S$. & 0 & 0,0 & 1 & 3,6 & 1 & 3,6 \\
\hline Trichuris trichura & 0 & 0,0 & 1 & 3,6 & 1 & 3,6 \\
\hline Larva Enterobius verniculares & 0 & 0,0 & 0 & 0,0 & 0 & 0,0 \\
\hline Total & 10 & 35,7 & 18 & 64,3 & 28 & 100,0 \\
\hline $\mathrm{p} \geq 0,05$ & & & & & & \\
\hline
\end{tabular}


Tabla 5. Frecuencia de parasitosis según bruxismo.

\begin{tabular}{lcccccc}
\hline & \multicolumn{3}{c}{ BRUXISMO } & \multicolumn{2}{c}{ Total } \\
& \multicolumn{2}{c}{ No } & \multicolumn{2}{c}{ Si } & & \\
& n & $\mathbf{\%}$ & n & \% & n & \% \\
\hline Parasitosis & & & & & & \\
Sin parasitosis & 20 & 31,3 & 44 & 68,8 & 64 & 69,6 \\
Con parasitosis & 12 & 42,9 & 16 & 57,1 & 28 & 30,4 \\
Total $\quad 32$ & 34,8 & 60 & 65,2 & 92 & 100,0 \\
$\quad \mathrm{p} \geq 0,05$ & & & & & & \\
$0,22<\mathrm{OR}<1,69$ & & & & & & \\
\hline
\end{tabular}

La mayor frecuencia de niños parasitados correspondió al sexo femenino: 18 niñas $(64.3 \%$ de 47 niños parasitados de ambos sexos) en tanto que al sexo masculino correspondió el $35.7 \%$ de 28 niños parasitados de ambos sexos. Por tipo de parásito, los más frecuentes fueron: Giardia Lamblia y Áscaris Lumbricoides. No se encontraron diferencias significativas entre presencia de parasitosis o entre tipo de parásito, según sexo (Tabla 4). Tampoco se halló asociación entre parasitosis y bruxismo (Tabla 5).

\section{DISCUSIÓN}

El bruxismo ocurre en todas las poblaciones desde edades tempranas hasta la adultez, con mayor prevalencia en los primeros años, declinando en la adolescencia y adultez. Y es de interés de varias disciplinas y especialidades de la salud, entre ellas, las relacionadas al dolor orofacial y a la medicina del sueño; y en odontología, de la odontopediatría y la oclusión $(1,3,4)$. Su presencia en niños puede persistir hasta la adultez relacionándosele con problemas de la articulación temporo-mandibular y problemas periodontales $(11,12)$.

La etiología del bruxismo es de naturaleza multifactorial. Principalmente, debido a factores periféricos como estímulo al sistema nervioso central y apretamiento dental, dando como respuesta el bruxismo. Algunas afecciones sistémicas, consideradas factores de riesgo son: problemas endocrinos y gastrointestinales, siendo la parasitosis gastrointestinal uno de los mas importantes (1315). Las infecciones parasitarias intestinales son de alta prevalencia en la población, especialmente en los países menos desarrollados; que incluyen a protozoarios $\mathrm{y}$ helmintos $\mathrm{y}$ causan numerosos síntomas, entre ellos bruxismo (21). Algunos reportes (12-21) mencionan también al Enterobius vermicularis vinculado con el bruxismo.

Aunque las prevalencias de bruxismo y parasitosis varían entre las poblaciones y regiones, éstos son considerados problemas importantes, por afectar a más del $10 \%$ de la población en distintas regiones. Así pues, las infecciones parasitarias intestinales constituyen un problema de salud pública por sus implicancias en la salud general y la calidad de vida de las poblaciones. Aunque, en algunos estudios $(15,25-27)$ no se encontró asociación estadísticamente significativa entre bruxismo y parasitosis intestinal, por su prevalencia en la niñez y su posible relación con alteraciones dentarias, periodontales y de la articulación temporo-mandibular, el bruxismo adquiere importancia en odontología.

La frecuencia de bruxismo encontrada en el presente estudio fue de $65 \%$, semejante a la encontrada en Buenos Aires (Morropón, Piura) y superior a la encontrado en otras poblaciones (59,22). La semejanza con el estudio en Buenos Aires (zona ubicada en el mismo departamento, pero en la Provincia de Morropón) se debería probablemente a la coincidencia de edades de los grupos estudiados; y las diferencias con otros estudios $(25,27)$ estarían relacionadas también con las edades de los pacientes evaluados. En el presente estudio la proporción de bruxismo en niñas fue mayor que la encontrada en niños; en tanto, que en el estudio realizado Buenos Aires (Morropón, Piura), niñas y niños tuvieron la misma proporción. En general, la literatura no reporta diferencias significativas en la prevalencia de bruxismo según sexo. Al analizar la presencia de bruxismo, según edad, el presente estudio encontró 
que éste fue más alto en los niños de 5 años, seguida de los de 4 y luego los de 6. Situación diferente a lo hallado en Buenos Aires (Morropón, Piura) donde las proporciones, de mayor a menor, fueron: 5, 6 y 4 años de edad, existiendo coincidencias sólo con la edad: 5 años.

La parasitosis intestinal en el presente estudio tuvo una frecuencia de $30 \%$ en niños de la muestra y la proporción en niñas fue casi el doble. Los parásitos más frecuentemente hallados fueron Giardia lamblia y Áscaris lumbricoides. No se encontró Enterobius vermicularis. En el estudio realizado en Buenos Aires (Morropón, Piura), las niñas tuvieron menor proporción de parasitosis que los niños. Las diferencias menores, en la correlación sexo según edad, en ambos estudios, podría ser debido a que a pesar de ser poblaciones de un mismo departamento, la población de La Brea (Talara, Piura) es periurbana y la de Buenos Aires (Morropón, Piura) es rural, lo que implica algunas diferencias en hábitos higiénicos y condiciones sanitarias.

Evaluando la posible asociación del bruxismo con parasitosis, se ha encontrado que niños con parasitosis tienen una proporción mayor de bruxismo $(57,1 \%)$. Pero entre los no parasitados, la proporción de niños con bruxismo es aún mayor $(68,8 \%)$. En el estudio de Buenos Aires (Morropón, Piura), la proporción de niños parasitados con bruxismo fue mayor $(81,8 \%)$ que la de niños sin bruxismo y la proporción de niños no parasitados con bruxismo fue menor $(56,3 \%)$. Así, en el presente estudio la proporcion de niños con parasitosis intestinal y bruxismo fue menor que en Buenos Aires (Morropón, Piura), pero en ambos estudios no se encontró asociación. Es de notar que en los niños de La Brea (Talara, Piura) no se encontró Enterobious vermicularis, mientras que entre los niños de Buenos Aires (Morropón, Piura), este parásito fue uno de los dos más frecuente.

Se concluye que en los niños de La Brea (Talara, Piura) la frecuencia de bruxismo fue alta, mayor en niñas que en niños y más alta en la población infantil de 5 años de edad. La parasitosis intestinal estuvo presente en 3 de cada 10 niños. No se encontraron diferencias significativas entre bruxismo y parasitosis.

\section{Correspondencia:}

Cindy Sutey Baldeón Sifuentes

Virgen de Fátima Mz. H Lt. 27 - San Martín de Porres. Lima, Perú.

Correo electrónico: cyndi.baldeon@upch.pe

\section{REFERENCIAS BIBLIOGRÁFICAS}

1. The glossary of prosthodontic terms. J Prosthet Dent 2005; 94 (1):10-92.

2. Attanasio R. Intraoral orthotic therapy. Dent Clin North Am. 1997; 41:309-24.

3. Okeson JP. Dolor orofacial: Guía para la evaluación, diagnóstico y manejo. 6ta. ed. Chicago: Editorial Quintessence; 1996.

4. American Academy of Sleep Medicine. International classification of sleep disorders. $2^{\text {a }}$ ed. Westchester; 2005

5. Nq DK, Kwok KL, Poon G, Chau KW. Habitual snoring and sleep bruxism in a paediatric outpatient population in Hong Kong. Singapore Med J. 2002; 43(11):554-6.

6. Cheifetz AT, Osganian SK, Allred EM, Needleman HL. Prevalence of bruxism and associated correlates in children as reported by parents. J Dent Child. 2005; 72(2):67-73.

7. Valera FC, Travitzki LV, Mattar SE, Matsumoto MA, Elias AM, Anselmo-Lima WT. Muscular functional and orthodontic changes in pre school children with enlarged adenoids and tonsils. Int $\mathrm{J}$ Paediatr Otorhinolaryngol. 2003; 67(7):761-70.

8. Delgado C. Relación entre el bruxismo y parasitosis intestinal en niños pre-escolares de 4 a 6 años de CEI N ${ }^{\circ} 301$ del Distrito de Buenos Aires, Provincia de Morropón. Departamento de Piura [Tesis pregrado]. Lima: Universidad Peruana Cayetano Heredia. Facultad de Estomatología; 2002.9.

9. Bader G, Lavigne G. Sleep bruxism; an overview of an oromandibular sleep movement disorder. Review article. Sleep Med Rev. 2000; 4(1):27-43.

10. Serra-Negra JM, Ramos Jorge ML, Flores-Mendoza CE, Paiva SM, Pordeus IA. Influence of psychosocial factors on the development of sleep bruxism among children. Int J Paediatr Dent. 2009; 19(5):309-17

11. Carlsson GE, Egermark I, Magnusson T. Predictors of bruxism, other oral parafunctions, and tooth wear over a 20-year follow-up period. J Orofac Pain. 2003; 17(1):50-7.

12. CamparisCM, Siqueira JT. Sleep bruxism: 
clinical aspects and characteristics in patients with and without chronic orofacial pain. Oral Surg Oral Med Oral Pathol Oral Radiol Endod. 2006; 101(2):188-93.

13. Koyano K, Tsukiyama Y, Ichiki R, Kuwata T. Assessment of bruxism in the clinic. J Oral Rehabil. 2008; 35(7):495-508.

14. Magnusson T, Egermarki I, Carlsson GE. A prospective investigation over two decades on signs and symptoms of temporomandibular disorders and associated variables. A final summary. Acta Odontol Scand. 2005; 63(2):99109.

15. Marks MB. Bruxism in allergic children. Am J Orthod. 1980; 77(1):48-59.

16. Gamboa MI, Kozubsky LE, Costas ME, Garraza M, Cardoso MI, Susevich ML, Magistrello NP, SavoneGT.Asociación entregeohelmintosycondiciones socioambientales en diferentes poblaciones humanas de Argentina. Rev Panam Salud Pública. 2009; 26(1):1-8.

17. Relman D, Mandell D. Enfermedades infecciosas: principios y prácticas. $5^{\mathrm{a}}$ ed. Buenos Aires: Editorial Médica Panamericana; 2002.

18. Murray PR, Rosenthal KS, Pfaller MA. Microbiología Médica. 5ta. ed. Madrid: Elsevier; 2006.

19. Berrocal N, Gracia H, Sánchez M. Parasitosis Intestinal y su relación con la calidad del agua y otros factores de riesgo en niños desplazados menores de 7 años, ubicados en el municipio de Montería Córdoba 2004.Universidad de Córdoba Facultad de Ciencias de la Salud Programas de Enfermería y Bacteriología. (Internet) 2010 (Citado el 15 de Marzo de 2010); 26(3). Disponible en: http:// www.academico.unicodoba.edu.co:8080.

20.Freeman B. Microbiología de Brurrows. $22^{\circ}$ ed. México DF: Interamericana/McGraw-Hill; 1989.

21. Pan American Health Organization, World Health
Organization. Helmintiasis Intestinales. Manejo de las Geohelmintiasis. Washinston DC: Pan American Health Organization, World Health Organization; 2003.

22. Ledesma A, Fernández, G. Enteroparasitosis: Factores predisponentes en población infantil de la Ciudad de Resistencia, Chaco. Universidad Nacional Noreste Comunicaciones Científicas y Tecnológicas. (Internet) 2010 (Citado el 23 de Marzo del 2010); 26(3). Disponible en: http://www. un n e. ed u . a r / W e b / c y t / c o m $2004 / 3$ Medicina/M-044.pdf.

23. Marcos L, Maco V, Terashima A, Samalvides F, Miranda E, Gotuzzo E. Parasitosis intestinal en poblaciones urbana y rural en Sandia, departamento de Puno, Perú. Parasitol Latinoam. 2003; 58(3):35-40.

24. Ahmad R. Bruxism in children. J Pedod. 1986; 10(2):105-26.

25. Tehrani MH, Pestechia N, Yousefi H, Sekhavati $\mathrm{H}$, Attarzadeh $\mathrm{H}$. The correlation between intestinal parasitic infections and bruxism among 3-6 year old children in Isfahan. Dent Res J. (Isfahan) 2010; 7(2):51-5.

26. Matta C, Balarezo A, del Castillo C, Evangelista A, Jara MJ, Delgado C. Asociación entre bruxismo y parasitosis intestinal en niños de 4 a 6 años de CEI No 301 de la localidad de Buenos Aires, Departamento de Piura, Perú 2001. Odontol Pediatr. 2005; 4(2):3-6.

27.Diaz-Serrano KV, da Silva CB, de Alburquerque S, Pereira Saraiva Mda C, Nelson Filho P. Is there an association between bruxism and intestinal parasitic infestation in children? J Dent Child . 2008; 75(3):276-9.

Recibido: 13/09/2013

Aceptado: 15/12/2013 